\title{
Electrical Power Management for Distributed Automotive System
}

\author{
X.F. Zhang, M.H. Luo, Y. Shen \\ Clean energy automotive engineering center \\ Tongji University \\ Shanghai, China
}

\author{
J.D. Cao \\ China Academy of Transportation Sciences \\ Beijing, China
}

\begin{abstract}
The electrical power management for distributed automotive system is proposed. The electrical power requirement is analyzed and the steady power source and electrical safe power source are independently proposed. Power supply channel with three-level current protection is designed to construct an electrical safe power supply which includes selective overload protection, fast short current protection and fuse backup protection. Prototype of electrical power management system is developed based on intelligent relay. The test result showed that the electrical management works well and the protection method is effective.
\end{abstract}

Keywords-electrical power management; steady power source; electrical safe power source

\section{INTRODUCTION}

With the rapid development of automotiveelectronictechnologies, electronic/electrical devices (EEDs) such asvehicle information systemshave become much more complicated than everbefore ( $\mathrm{Gu}$ et al. 2010), at the same time more and more optimization work were carried out to simplify harness wires in automotive system(Zhang \& Shen 2014). Available surveys (Toerngren et al. 2008, Giovanni et al. 2005)revealed that the embedded control with network connections, such as CAN (Control Area Network), LIN (Local Interconnection Network), has become a trend.At the same time, more and more intelligent EEDs come into reality in Automotive, which have over current protection and self-diagnostic function (Russell 2005). In such asystem, the wires which transfer digital signals can be separated from the wires that transfer the electrical power, inducing the independence of communication network and electrical power network, which facilitates the electrical power distribution and management design.

In this paper, an electrical power management method,which is suitable for distributed electrical system in automotive is proposed.Three-level protection method, includingsoftware overload protection, fast short current protection and fuse backup protection, are adopted to meet over current protection requirement. Electrical power management system (EPMS) and its prototypeis developed and tested,which is proved to be effective and came into usage.

\section{Distribution of Automotive Electrical Power SUPPLY}

\section{A. Electrical Power Requirement}

The requirements for electrical power can be divided into two types. First, the power forelectricaldevices,such as electrical motors, electrical heaters, electrical vales and lights, etc. The voltage is always $12 / 14 \mathrm{~V}$ in passenger cars and $24 / 28 \mathrm{~V}$ in trucks or buses. Second, the power for circuits in ECU (Electric Control Unit) and communication network. The circuits inside an ECU usually works under 5 or $3.3 \mathrm{~V}$, sometimes $2.5 \mathrm{~V}$ or even lower. Astabilized power of $8-18 \mathrm{~V}$ is also asked by LIN bus system as a reference voltage.

System voltage perturbation appears frequently when the electronic circuits are instable, eg.at the starting time by the starter, and sometimes by the burst into usage of high power devices or cutoff of inductive loads while vehicle's working. System voltage perturbation could cause digital communication problems, such as CAN error frames and LIN communication errors.

In general, in a distributed automotive electrical system, an electrical-safe power source (ESPS) as well as stable-neat power source (SNPS) is independently necessaryfordifferent requirements. The former is for lower voltage stability and higher energy consumption, and the latter provides an uncontaminated stable power source and lower energy consumption.

\section{B. System Layout}

A typical electrical system is as Figure 1 shows (Gu et al. 2010).d and $\delta$, namely the intelligent EEDs with CAN and LIN digital interface, are composed of the original EEDs and an onsite ECU. The intelligent EEDs are networked and organized into one system, which features a hierarchical structure. The $\delta$ s are firstly grouped as several local networks (L in Figure 1) according to a certain criteria, e.g. function similar criteria, location adjacent criteria, etc. secondly Ls and ds are connected through gateways. Finally, Ls and ds together compose the system. 


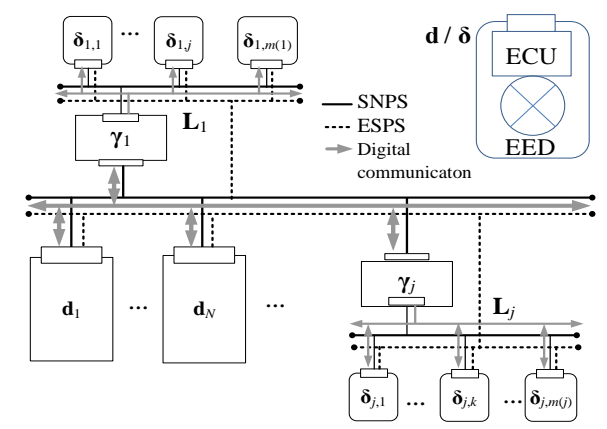

FIGURE I. DISTRIBUTED ELECTRICAL SYSTEM LAYOUT.

As referred in last section, SNPS and ESPS compose an independently electrical power network in an electrical power supply. SNPS is usually composed of a constantly-on-source part and aswitchable part. Some EEDs need slight electrical power in a parking vehicle, e.g. remote sensor, clock, memory inside odometer and instrument to keep the number of miles and individual configuration, anti-thief system, keyless entrance system, etc. The EEDs mentioned above need sustained but much limited power supply, e.g.10mA@12V or even lower. In contract, most of electrical circuits inside ECUs are shut down while parking, when there is no consumption and energy requirement.

So normal-open SNPS andswitchable SNPS are figured out. The former is to power the constantly working EEDs while the latter is for all other ECUs'and to minimize the transient current during parking, which has a great effect on battery's state of charge (SOC).

\section{Electrically Safe Power Source}

The ESPS network is composed of several power supply channels (PSCs). PSC is a switchable electrical transfer wire with over current protection. Grounding points are necessary for less wires and connectors. A high power volume EED has its dedicated PSC, e.g. starter, while several EEDs in the adjacent area or same local network share one PSC to reduce the harness complexity. It is the problem to keep electrical safety after the removal of traditional fuse box. The EPMS is introduced for PSC current protection as well as battery SOC monitoring.

\section{Three-LeVel over CurRent Protection}

\section{A. Current Protection of Power Supply Channel}

Three-level over current protection is proposed to ensure PSC's electrical safety. The current protection points A C along the PSC are as Figure 2 shows.

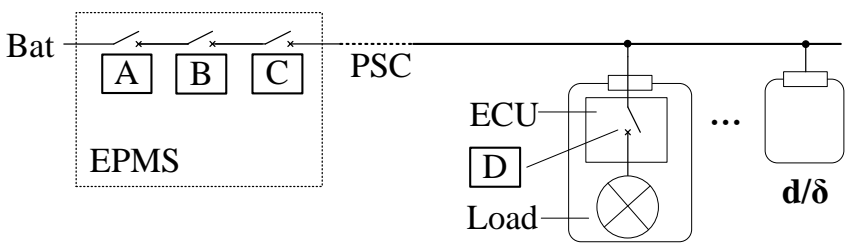

FIGURE II. PROTECTION POINTS OF POWER SUPPLY CHANNEL.

As shown in Figure 2, "A" is software over current protection based on MCU (Micro-Control Unit).The time delayed before current break is calculated according to the magnification of the actual current to the allowed maximum. Itobtains the tolerance of current pulse and make it flexible(Gu\& Li 2005). In most case, protection will be activated at point D as the PSC should not be broken down frequently because it is responsible to several EEDs. As a result, time delay function and current pulse tolerance are necessary. "B" is fast break-out protection in case of short circuit, e.g. PSC wire short to ground. "C" is arecoverable fuse as backup protection. It will cut off the PSC in case of "A" and "B" failure. " $\mathrm{P}$ " is intelligent power electronics inside the on-site ECU of $\mathrm{d} / \delta$ rather than a traditional fuse. And A $\sim \mathrm{C}$ are set for the possible failure at point " $\mathrm{D}$ ".

\section{B. Software Current Protection}

Nowadays, intelligent circuit breaker, three-segment protection method is adopted (Kosack 1999), namely the long time delay, short time delay over current protection and short circuit protection, is used to obtain excellent tolerance.

The real time current or normal current IR equals to the sum of each running EEDs' normal power divided by nominal system voltage. Then the nominal delay time features proportion to the magnification factor $\mathrm{K}$. The relation between action time $\mathrm{T}$ and $\mathrm{K}$ can be as in Equation (1):

$$
\left\{\begin{array}{c}
K T=K_{1} t_{1} \quad K_{1}<K<K_{2} \\
K T=K_{2} t_{2} \quad K_{2}<K
\end{array}, K=I_{R} / I\right.
$$

where, I= the real time current from the sensor; $\mathrm{K} 1=$ the lower limit of long time delay protection over current factor; $\mathrm{K} 2=$ the lower limit of short time delay protection over current factor; $\mathrm{t} 1, \mathrm{t} 2$ = the action time under $\mathrm{K} 1$ and $\mathrm{K} 2$.

The software current protection is implemented by MCU. As is shown in Figure 1, all the EEDs' status can be monitored and the reference current on a specific PSC can be obtained with the additional rated power of the working EEDs. An individual current sensor is laid on PSC and the real time over current factor ki can be achieved at each sample time.

Left time of protection $\tau$ is defined as Equation (2):

$$
\tau_{i}=\left\{\begin{array}{cc}
\tau_{i-1}+t_{R} & k_{i}<K_{1} \\
\tau_{i-1}-\frac{T_{i}}{T_{i-1}} T_{s} & k_{i}>K_{1}
\end{array}\right.
$$

where, $\tau \mathrm{i}$ and $\tau \mathrm{i}-1=$ the left time at the ith and (i-1)th sample time; Ti, Ti-1 = the action delay time according to Equation (2); $\mathrm{tR}=$ the recoverable factor;Ts $=$ MCU's sample time. $\tau$ has its up limit of Tu, which is also the initial value of $\tau$. When $\tau \leqslant$ 0 , the PSC will be cut off and the software current protection happens.

\section{Fast Break-out Protection}

In case of short circuit current on PSC, which exceed the PSC's maximum allowed current, a fast break out protection should be taken place. Software protection cannot react within one millisecond with a slow 8 bit MCU. For that a circuit for fast break out is designed. As Figure 4 shows, U1 is from the current sensor and U0 is the reference voltage, namely the maximum allowed current on PSC. In case of the short circuit current on PSC, U1 is higher than U0, the comparator's output 
will be zero and the $R \& D$ trigger will give out a low voltage level on Q terminal, too. So second coil powered by the MOSFET will be shut down because of the gate electrode voltage drop, thus the relay is broken out.

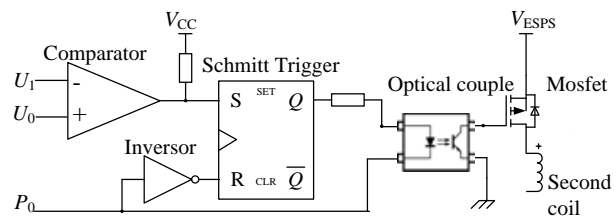

FIGURE III. FAST BREAK-OUT CIRCUIT.

After the clearance of short current status sensor voltage U1will drop and the comparator output will be recovered to the TTL high voltage. Although, thanks to status lockage by the $\mathrm{R} \& \mathrm{D}$ trigger, the output of $\mathrm{Q}$ terminal remains lower, and the MOSFET remains closed, thus keeps the relay closed. R\&D trigger can be reset through port P0 by MCU.

The delay time of the fast break out protection consists of:

- Response time of Hall sensor, equal to $\mathrm{O}\left(10^{-6}\right) \mathrm{s}$;

- Delay by first order transfer function of RC filter. The delay can be calculated as Equation (3):

$$
t_{R C}=-T_{R C} \cdot \ln \left(1-\frac{1}{K}\right)
$$

- where, TRC= the RC constant; $\mathrm{K}=$ theratio of current magnitude to maximum allowed current of PSC, and $\mathrm{K}$ is always more than 1 .

- Response time of R\&D trigger, equal to $O(10-9) s$;

- Response time of optical couple, about $50 \times 10-6 \mathrm{~s}$;

- Response time of MOSFET, equal to O(10-9)s;

- Response time of Relay, about $2 \times 10-3$ s.

- According to analysis above, the total time delay can be within 3 milliseconds. Thus the delay can be equal to $\mathrm{O}(10-6) \sim \mathrm{O}(10-3) \mathrm{s}$.

\section{Back up Fuse Selection}

Backupfusemay work in case of failure of fast break out protection and software protection. It is very important to make sure that backup fuse wouldn't act before the software protection or fast break out protection. Protection curve of backup fuse should be selected compared with that of time delay protection. The dashed line is obtained from Equation (3) and reasonable margin between the two lines. So the backup fuse's time delay protection curve should always be above that of the software protection.

\section{Power Management System Prototype}

\section{A. System Layout}

In the EPMS, each PSC is controlled by IRs (Intelligent Relays), while the number of IRs depends on system requirement. The gateway (GW) is used to generate the SNPS as well as to transfer digital signals between the local network and its superior. The BMU (Battery ManagementUnit) monitors the total current consumed. System layout is as shown in Figure 4.

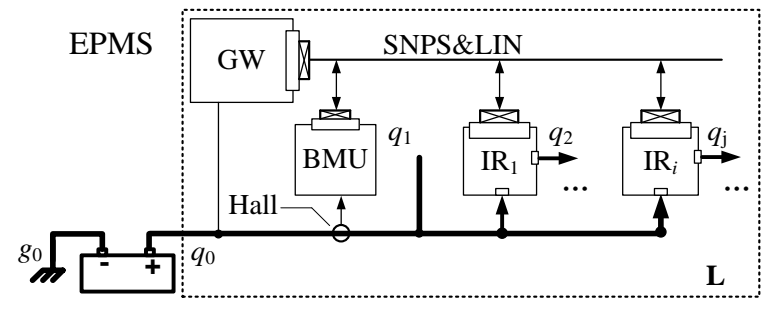

FIGURE IV. EPMS SCHEMATIC ANDLAYOUT.

Contact-type relays were chosen and the schematic of IR is designed. LIN interfacecircuit and relay contact diagnostic circuit are also designed except the fast break-out circuit mentioned above. The controllers of IR are designed and implemented. The EPMS system was also developed as one assembly for a travel bus electrical system, as shown in Figure 5.

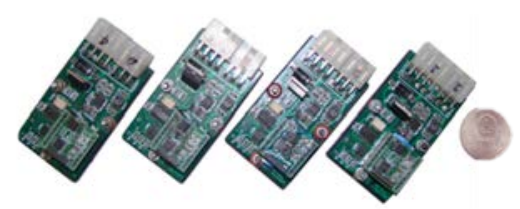

(A)

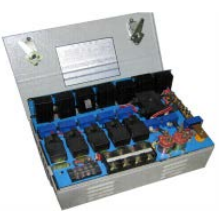

(B)
FIGURE V. IR'S CONTROLLER AND EPMS PROTOTYPE.

\section{B. Over Current Protection Test}

The CAN bus test tool Canalyzer was used to simulate the digital communication network. Gateway was also used to set the reference current and the actual load was performed by electrical load. The software protection test process is as Figure 6 shown.



FIGURE VI. SOFTWARE PROTECTION TEST.

K1 were set to 1.15 and $\mathrm{K} 2$ were 2 . Andt1, t2 were set to140 and 90s. Four segments were performed:

- the reference current was set to $8 \mathrm{~A}$ and the load was about 10A. EPMS worked well and no shut down warning signal was sent;

- the actual load was set to about $12 \mathrm{~A}$, so slight over load happened. After around 73 seconds, "Channel will shut down in 60 seconds" warning was sent out and recorded by Canalyzer; 
- the actual load was set to about 30A, so serious over load happened. After around 80 seconds, PSC was closed;

- the state of IR was reset and a current of 3A is loaded. The IR and PSC recovered.

The proposed electrical power distribution method as well as the EPMS has been applied to a prototype vehicle, a midsize concept car and a twelve-meter long city shuttle bus.

In total of 10 PSCs are created to power more than 100 EEDs. More than twenty thousand kilometers road test was done and it was proved to be safe and reliable.

\section{CONCLUSIONS}

(1) Steady-neat power supply and electrical safety power supply can fulfill the electrical power requirement of distributed electrical system in automotive.

(2) Power supply channel based electrical power distribution method is feasible. Software over current protection obtain good flexibility, fast break out protection is also work well.

(3) The three-level current protection method can ensure electrical safety in automotive system.

\section{ACKNOWLEDGMENT}

This work was supported by the and National High Technology Research and Development Program of China (2013AA12A026)and National Instrument Special Funding on Automotive fuel cell system testing (2012YQ150256).

\section{REFERENCE}

[1] Christopoulos, C. \&Wright, A. 2005.Electrical power system and its protection. Beijing: electrical power press of China.

[2] Giovanni, T., Joseph, N. \& Gary, B. 2005. Evolutionand trends in automotive electrical distributionsystems. In Proceedings of the IEEE Vehicle Powerand Propulsion Conference: 812-818.

[3] Gu, S.Q. \& Li, F.R. 2005.Electrical power system and its protection. Beijing: electrical power press of China.

[4] Gu, Z.M.,Yang, D.G. \&Zhang, X.F. 2010. Distributed vehicle body electric/electronic system architecture with central coordination control. International journal of automotive engineering 224(2): 189-199.

[5] Kosack, K. 1999. Low voltage electrical device and switch datasheet: a select criteria and design guide.Beijing:China Machine Express.

[6] Russell, M.E. 2005. Integrated automotive sensors.IEEE Transaction on Microwave Theory andTechniques 50(3): 674-677.

[7] Toerngren, M., Johansson, K.H., Andersson, G.,Bodin, P. \&Purdue, D.2008. Assessment of high-integrity embedded automotive control systems using hardware in the loop simulation. Journal of Systems and Software 81(7): 1163-1183.

[8] Zhang, X.F. \& Yong, S. 2014. Networking optimization for Holodistributed automotive body electrical system. Journal of computers 9(1): 112-117. 